\title{
PEPA: Entwicklung eines Scoring-Modells zur Priorisierung von Prozessen für eine Automatisierung
}

\author{
Ralf Plattfaut $\mathbb{D} \cdot$ Julian F. Koch $\cdot$ Michael Trampler $\cdot$ André Coners
}

Eingegangen: 29. Mai 2020 / Angenommen: 3. Oktober 2020 / Online publiziert: 16. Oktober 2020 (C) Der/die Autor(en) 2020

Zusammenfassung Robotic Process Automation (RPA) bezeichnet eine Technologie, die die einfache Erstellung von Computerprogrammen (sogenannten Bots) zur Automatisierung von IT-gestützten Geschäftsprozessen über die graphische Benutzeroberfläche ermöglicht. Aktuelle Forschungsbemühungen im Themenfeld RPA haben gezeigt, dass der erfolgreiche Einsatz von RPA allem voran ein realistisches Erwartungsmanagement und eine ausgiebige Prozessaufnahme erfordert. Diese Resultate zeigen die Notwendigkeit von Bewertungsmethoden zur Bestimmung und Klassifizierung von Prozessen im Hinblick auf ihre Verwendbarkeit im Rahmen von RPA.

In der Arbeit werden Ergebnisse eines mehrstufigen Design-Science-ResearchProjekts im Kontext eines mittelständischen Industrieunternehmens vorgestellt. Genutzt werden dafür strukturierte mehrstufige qualitative Expertenbefragungen mit dem Ziel der Modellbildung. In diesem Projekt wird demnach ein neues Bewertungsmodell zur Messung der Eignung für eine RPA-Implementierung und eine detailliertere Potenzialanalyse entwickelt (PEPA, Prozesseignung und -Priorisierung für Automatisierung). Das PEPA-Modell, von der Konzeptualisierung bis zur Implementierung, konzentriert sich auf seine verallgemeinerbare Anwendung und bietet ein systematisches Vorgehen zur Eignungsanalyse von Prozessen. Es berücksichtigt dabei wirtschaftliche, technologische und prozessuale Kriterien und ermöglicht eine anschließende Priorisierung der Prozesse in Bezug auf ihre Eignung für eine RPA-

\section{R. Plattfaut $(\bowtie)$}

Process Innovation and Automation Lab, Fachhochschule Südwestfalen, Lübecker

Ring 2, 59494 Soest, Deutschland

E-Mail: plattfaut.ralf@fh-swf.de

J. F. Koch · M. Trampler · A. Coners

Fachhochschule Südwestfalen, Fachbereich Technische Betriebswirtschaft, Haldener

Straße 182, 58095 Hagen, Deutschland 
Implementierung. Damit geht das vorgeschlagene PEPA-Modell über bestehende Modelle aus der Praxis hinaus.

Schlüsselwörter Robotic process automation - Scoringmodell · Fallstudie · Angewandte Forschung · Design Science Research

\section{PEPA: Development of a Scoring Model to Prioritize Business Processes for Automation}

Abstract Robotic Process Automation (RPA) is a technology that allows the easy development of computer programs (i.e., bots) which automate computerized business processes through the usage of Graphical User Interfaces. Current publications show that successful RPA implementations especially require a realistic management of stakeholder expectations and an in-depth process analysis. These results underline the need for decision support models to estimate impact of RPA on business processes, and, as such, prioritize business processes for RPA implementation.

In this article we present results of an iterative Design Science Research project. The project was conducted in the context of a medium-sized industrial company. The overall aim is to create and evaluate a new scoring model for the prioritization of business processes for automation. The created model offers systematic support in evaluating different processes, ranks these processes with regards to monetary, technological and process-oriented criteria and, thus, allows a prioritization of processes.

Keywords Robotic process automation - Scoring model $\cdot$ Case study · Applied research $\cdot$ Design science research

\section{Einleitung}

Automatisierung von Geschäftsprozessen hat einen enormen Einfluss auf die Effizienz in Unternehmen. In einer Studie des McKinsey Global Institute wird postuliert, dass bis zu 50\% aller heute ausgeführten Tätigkeiten automatisiert werden können (Manyika et al. 2017). Die Untersuchung von Prozessautomatisierung in der Wirtschaftsinformatik ist nicht neu. Van der Aalst und Kollegen nennen die Automatisierung von Geschäftsprozessen eine grundlegende Frage der Wirtschaftsinformatik (van der Aalst et al. 2018b). In der Tat sind in den vergangenen Jahrzehnten durch Produktionsplanungs- und steuerungssysteme, Warenwirtschaftssysteme, Enterprise Resource Planning (ERP) Systeme und weitere Technologien wie Workflow Management oder Business Process Management (BPM) Systeme hohe Anteile von heute ablaufenden Prozessen automatisiert worden (Sumner 2005; Mohapatra 2009; van der Aalst et al. 2018b).

Robotic Process Automation (RPA) ist ein vergleichsweise neuer, leichtgewichtiger Ansatz zur Automatisierung von Geschäftsprozessen (Lacity und Willcocks 2016; Øvrelid und Halvorsen 2018; Plattfaut 2019). Auch wenn es noch keine einheitliche Definition von RPA gibt, ist doch klar, dass ein Kernbestandteil von RPA 
die Automatisierung von Geschäftsprozessen durch einfach zu erstellende SoftwareRoboter (Bots) ist, die die Graphische Nutzerschnittstelle (Graphical User Interface, GUI) von existierenden Systemen bedienen. Diese Bots imitieren so die Interaktion von Nutzern mit Anwendungssystemen und ermöglichen eine Automatisierung ohne Veränderung der zu automatisierenden Anwendungssysteme. Dadurch können mit RPA Automatisierungslösungen für Geschäftsprozesse schneller und kostengünstiger entwickelt werden, als mit traditionellen Methoden (Penttinen et al. 2018; Lacity und Willcocks 2016; Mendling et al. 2018; Czarnecki et al. 2019).

Grundsätzlich sind verschiedene Prozesse zum Einsatz von RPA geeignet. Die Literatur stellt hier insbesondere repetitive und regelbasierte Prozesse in den Vordergrund (van der Aalst et al. 2018b; Hofmann und Günther 2019; Czarnecki et al. 2019). Gleichzeit scheint auch klar, dass mit Hilfe von RPA die Automatisierung von bisher vernachlässigten Prozessen lohnenswert erscheint (van der Aalst et al. 2018b, 2018a; Plattfaut 2019). Hierdurch kommen in Unternehmen eine Vielzahl von möglichen Prozessen in Frage. Die aktuelle Forschung stellt dabei heraus, dass noch ungeklärt ist, ,,welche Charakteristiken einen Prozess geeignet für RPA-Unterstützung machen“" (van der Aalst et al. 2018b, S. 271).

Dieser Artikel berichtet von einem praxisnahen Forschungsprojekt zur Entwicklung des Scoring-Modells PEPA (Prozesseignung und -Priorisierung für Automatisierung) für genau diese Fragestellung. Mit PEPA kann die Eignung von Prozessen für eine RPA-Implementierung vergleichend gegenübergestellt und eine erste Priorisierung der Prozesse für eine RPA-Implementierung ermittelt werden. PEPA berücksichtigt dabei wirtschaftliche, technologische und prozessuale Kriterien und ermöglicht eine anschließende Priorisierung der Prozesse in Bezug auf ihre Eignung für eine RPA-Implementierung. Dieses Vorgehen ist angelehnt an etablierte Verfahren zur Entscheidungsfindung und entsprechende Vergleichsmethoden (Wei et al. 2005). Das PEPA-Modell wurde dem Design-Science-Research-Ansatz (Hevner et al. 2004) folgend in verschiedenen Build-/Evaluate-Zyklen in enger Zusammenarbeit mit einem Industrieunternehmen entwickelt und getestet.

Der verbleibende Artikel ist wie folgt strukturiert: Zuerst wird das Themenfeld RPA genauer erläutert. Danach wird auf Scoring-Modelle als Gegenstand des Design Science Research eingegangen. Das konkrete Forschungsdesign und der Fallstudienhintergrund werden kurz erläutert. In Abschn. 5 werden die Ergebnisse aus der Entwicklung und Anwendung von PEPA vorgestellt. Der Beitrag schließt mit den Schlussfolgerungen, Implikationen für Forschung und Praxis sowie möglichen anschließenden Forschungsfragen.

\section{Robotic Process Automation}

Wie in der Einführung erläutert, ist die Optimierung und Automatisierung von Geschäftsprozessen eines der Kerngebiete der Wirtschaftsinformatik (van der Aalst et al. 2018b). Die Automatisierung von Geschäftsprozessen hat eine direkte Verbindung zu den aktuellen Digitalisierungsherausforderungen von Unternehmen, die beispielsweise unter dem Schlagwort ,Arbeitswelt 4.0“ oder ,Digitale Transformation“ behandelt werden (Meier et al. 2019; Niesen et al. 2019; Hofmann und Günther 
2019; Czarnecki et al. 2019). Dabei reicht die Automatisierung von Geschäftsprozessen von der einfachen Automatisierung von einzelnen Aufgaben oder Aktivitäten bis hin zur Automatisierung von vollständigen Ende-zu-Ende-Geschäftsprozessen (Dumas et al. 2018).

In den vergangenen Jahrzehnten wurden Geschäftsprozesse auf vier verschiedene Arten automatisiert (Mohapatra 2009; Penttinen et al. 2018): Erstens werden Kernanwendungssysteme, z. B. ERP-Systeme, um regelbasierte automatisierte Verarbeitung von Anwendungsfällen erweitert. Zweitens werden BPM-Systeme zur automatischen Steuerung von Prozessen eingeführt. Drittens können mit Hilfe von Middleware-Systemen verschiedene Anwendungssysteme über definierte Schnittstellen verknüpft werden. Viertens werden spezialisierte Werkzeuge zur Automatisierung von klar definierten Anwendungsfällen eingesetzt.

Im Gegensatz zu diesen eher traditionellen Methoden der Geschäftsprozessautomatisierung ist RPA ein leichtgewichtiger Ansatz, der es erlaubt, ohne Eingriffe in bestehende Anwendungssysteme Prozesse zu automatisieren (Lacity und Willcocks 2016; Plattfaut 2019). Dabei werden mit Hilfe von RPA-Systemen sogenannte Bots erstellt, die über die Präsentationsschicht/GUI existierende Systeme bedienen. Diese Bots imitieren die Interaktion von Nutzern mit den Anwendungssystemen (Penttinen et al. 2018; Lacity und Willcocks 2016; Mendling et al. 2018). Hierbei werden Bots hauptsächlich in regelbasierten und repetitiven Prozessen eingesetzt (Czarnecki et al. 2019).

Die Besonderheit bei RPA ist die einfache Erstellung der Bots weitestgehend ohne manuelle Programmierung. RPA-Systeme ähneln Skripten, Screen-Scraping oder Makros (Czarnecki et al. 2019) und greifen auf vorgefertigte Standardbausteine zurück. Die Erstellung von RPA Bots gilt dementsprechend als einfach, benötigt wenig Fachwissen und wird als No-Code oder Low-Code bezeichnet (Lacity und Willcocks 2016).

Durch diese einfache Erstellung ist RPA für die Automatisierung einer zweiten Welle von Prozessen geeignet. Während durch die oben erwähnten, eher traditionellen Ansätze vor allem jene Prozesse automatisiert wurden, die bei maximaler Häufigkeit der Prozessdurchführung großen Wert für das Unternehmen generieren (van der Aalst et al. 2018b), wird durch RPA die Automatisierung von Prozessen wirtschaftlich, die bisher aufgrund der geringeren Häufigkeit unwirtschaftlich war (van der Aalst et al. 2018b; Penttinen et al. 2018). Van der Aalst und Kollegen verdeutlichen diesen Zusammengang graphisch (vgl. Abb. 1): In der Abbildung sind schematisch alle Aufgaben einer Organisation in absteigender Häufigkeit der Durchführung abgetragen. Die häufig auftretenden Aufgaben sind in vielen Fällen schon durch traditionelle Methoden der Automatisierung angegangen. In den weniger häufig auftretenden Aufgaben ist eine traditionelle Prozessautomatisierung zu teuer. Hier kann mit RPA jetzt der mittlere Bereich der Aufgaben angegangen werden. Dies sind Aufgaben, die eine mittlere Häufigkeit haben und wo sich ein entsprechender RPA-Einsatz rentiert. Es verbleiben jedoch weiterhin Aufgaben, die von Menschen durchgeführt werden (van der Aalst et al. 2018b, 2018a).

Während die Identifikation der häufigsten Aufgaben in Unternehmen vergleichsweise einfach ist, fällt gerade im mittleren Bereich die Auswahl und Priorisierung für RPA häufig schwer. Die obige schematische Darstellung berücksichtigt darüber 


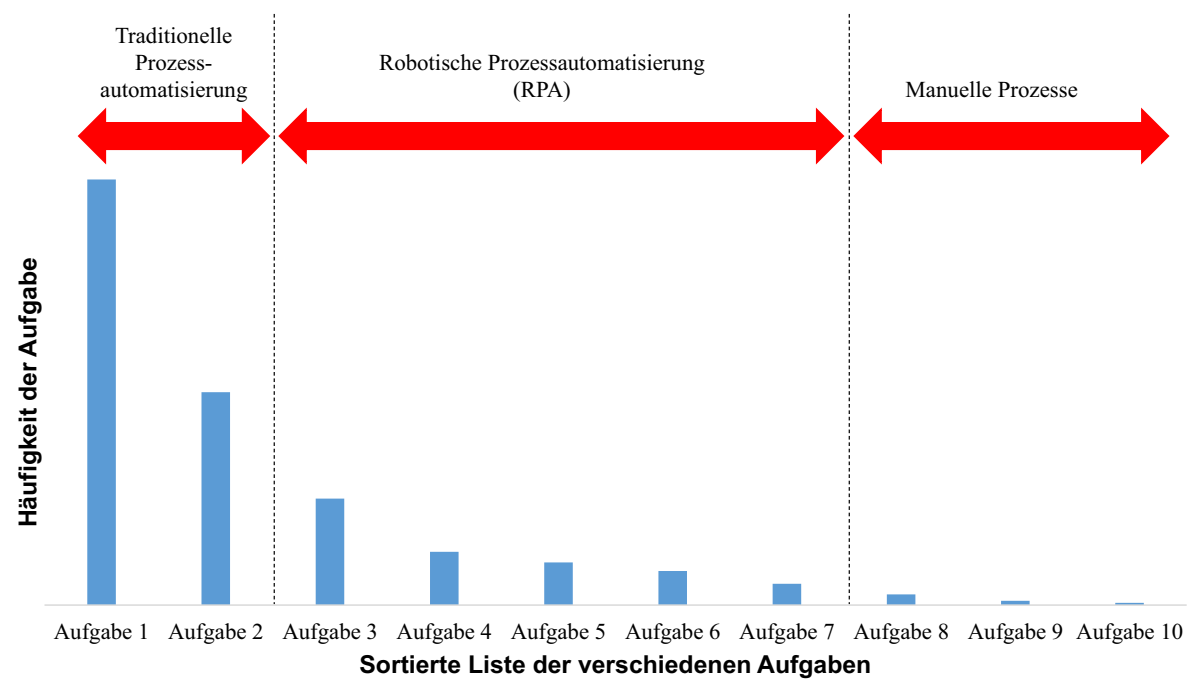

Abb. 1 Schematische Darstellung von Aufgaben, deren Häufigkeiten und einer Eignung für Automatisierung (vgl. van der Aalst et al. 2018b)

hinaus nur die Häufigkeit der Aufgaben. Andere Autoren fordern aber auch, dass in dieser Entscheidungssituation Effektivitäts- und Effizienzziele sowie nichtmonetäre, intangible Nutzeffekte Berücksichtigung finden müssen (Czarnecki et al. 2019). Es bedarf eines Priorisierungsmodells zur Identifikation geeigneter Aufgaben und Prozesse für die Automatisierung mit RPA (van der Aalst et al. 2018b; Czarnecki et al. 2019).

\section{Scoring-Modelle als Gegenstand des Design Science Research}

Scoring-Modelle sind als ein Erkenntnisgegenstand der Wirtschaftsinformatik etabliert. So wurden beispielsweise Scoring-Modelle zur Auswahl von geeigneten ITProjekten entwickelt (Muralidhar et al. 1990; Gerogiannis et al. 2010). Grundsätzlich sind Scoring-Modelle, zum Beispiel in Form der Nutzwertanalyse, auch für die Auswahl von zu beschaffenden Anwendungssystemen geeignet (Bensberg 2019). In den jüngeren Jahren wurden aus der Forschung heraus häufig Reifegradmodelle zur Bewertung von spezifischen Situationen und Unternehmen und zur Entsprechenden Präskription von Entwicklungspfaden entwickelt (Becker et al. 2009; Poeppelbuss et al. 2011).

Design Science Research ist ein gestaltungsorientiertes Forschungsparadigma zur strukturierten Entwicklung von Artefakten, die auf wissenschaftliche Theorien aufbauen und real existierende Probleme lösen (Becker et al. 2020; Hevner et al. 2004). Dabei besteht die Forschung aus verschiedenen Build-/Evaluate-Zyklen. In diesen Zyklen werden aufbauend auf konkreten Fragestellungen von Organisationen (Relevanz der Forschung) sowie wissenschaftlichen Grundlagen, Theorien und Methoden (wissenschaftlicher Rigour) Artefakte entwickelt und evaluiert. Aufbauend auf den 
Evaluationsergebnissen können dann die entwickelten Artefakte wieder weiterentwickelt werden, womit der nächste Build-/Evaluate-Zyklus startet. Zur Evaluation der Artefakte können neben analytischen Überlegungen auch andere empirische Verfahren wie Fallstudien oder Feldstudien eingesetzt werden (Hevner et al. 2004).

Die Untersuchung und Entwicklung von Scoring-Modellen im Design Science Research hat Tradition in der nationalen und internationalen Wirtschaftsinformatik (Becker et al. 2009; Mettler 2011). Dabei werden Scoring-Modelle aufbauend auf bestehenden wissenschaftlichen Veröffentlichungen entwickelt und dann in der Realität in Fallstudien getestet. Die Erkenntnisse der Fallstudie tragen dann zu einer Verfeinerung des Scoring-Modells bei. Ein ähnlicher Ansatz wird in dem hier vorliegenden Beitrag durchgeführt.

\section{Forschungsdesign}

Das hier vorgestellte Forschungsprojekt zur Entwicklung des Scoring-Modells PEPA folgt dem Design Science Research-Ansatz (Hevner et al. 2004). Mit dem PEPAModell kann die Eignung von Prozessen für eine RPA-Implementierung transparent beurteilt und eine erste Abschätzung der Priorisierung der Prozesse erstellt werden. In diesem konkreten Fall startet die Entwicklung mit empirisch gesammelten Evaluationsergebnissen aus der Anwendung eines existierenden Priorisierungsmodells eines Beratungsunternehmens in der Praxis. Darauf aufbauend werden iterativ vier der oben dargestellten Build-/Evaluate-Zyklen durchlaufen (vgl. Abb. 2).

Zur Entwicklung des Scoring-Modells wird ein integriertes DSR-Team aus Praktikern und Hochschulangehörigen gebildet. Dieses DSR-Team übernimmt die Evaluation und Weiterentwicklung des Modells. Iterativ wird das Modell von Anwendern getestet - die Ergebnisse dieser Anwendungen dienen dann zusammen mit weiteren Daten als Grundlage für den nächsten Evaluationsschritt des DSR-Teams. Details zu den einzelnen Entwicklungsschritten finden sich in Tab. 1.

Die Praktiker und Anwender sind dabei Mitarbeiter eines Unternehmens im Bereich der Metallverarbeitung. Das untersuchte Unternehmen beschäftigt weltweit 800 Mitarbeiter und produziert jährlich mehr als 500.000 Tonnen gewalzte Stahlbänder und -profile an Standorten in Europa, Nord- und Südamerika und Asien.

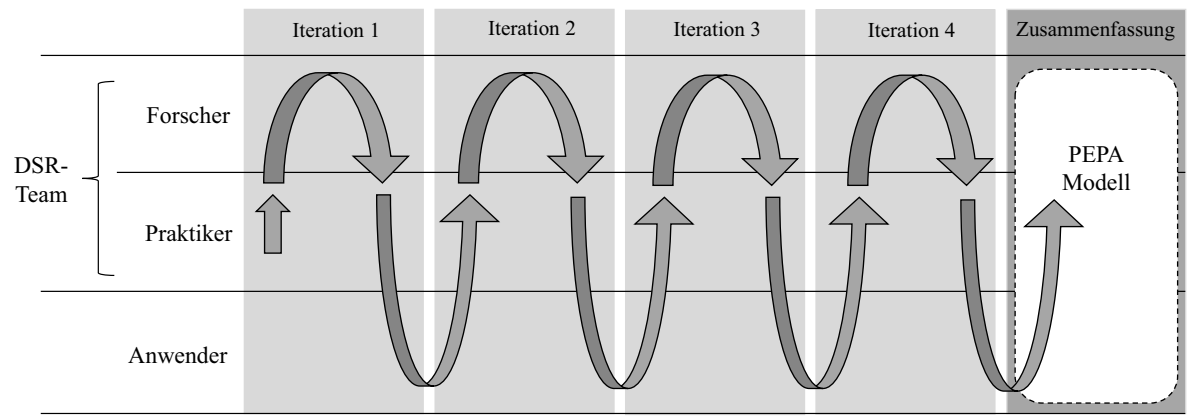

Abb. 2 Build-/Evaluate-Zyklen zur Entwicklung des PEPA-Modell in Anlehnung an Sein et al. (2011) 
Tab. 1 Entwicklungsschritte des Forschungsprojekts

\begin{tabular}{lll}
\hline Entwick- Aktivitäten & Ergebnis \\
lungs- & & \\
schritt & &
\end{tabular}

Anwendung - Eigenständige Anwendung eines bestehenden Modells zur eines Priorisierung aus einem Beratungsunternehmen durch das beste- Praxisunternehmen

henden

Modells

Evaluate I - Evaluation des bestehenden Modells und Ableitung von Ex-Ante-Anforderungen durch gemeinsamen Workshop mit Wissenschaftlern und Mitarbeitern des Praxisunternehmens (Controller Geschäftsbereich Services, Head of Finance and Controlling)

Build I - Erstellung eines ersten Prototypens für PEPA (PEPA 0.1) aufbauend auf den Evaluationsergebnissen sowie Erkenntnissen aus der Literatur

Evaluate II - Anwendung von PEPA 0.1 auf 16 Prozesse (u. a. Validierung von Verbrauchsstellen, Abrufen intervallgemessener Energieverbräuche, Abrechnung und Abrechnungsgenehmigung)

- Analyse von Kopfdaten von 36 Abschluss- und Zwischenberichten vergangener Prozessoptimierungen zur Vorbereitung der Experteninterviews und Identifikation von Merkmalsausprägungen. Kopfdaten enthielten Information zu Fachbereich, Laufzeit, Projektkategorie enthielten und wurden durch Daten zu Arbeitspaketen, Ressourceneinsatz, Abweichungen/ Qualitätsschlussfolgerungen angereichert. Analyse erfolgt sowohl qualitativ im Forscherteam und automatisiert durch Text Mining und Topic Modeling

- Durchführung von 16 semistrukturierten Experten-Interviews mit Anwendern von PEPA 0.1

- Analyse von 672 Standardprotokolldateien zur Ex-post-Evaluierung der zugrundeliegenden Prozesse. Diese Standardprotokolle werden automatisch bei Start und Beendigung sowie Auftreten von Systemfehler durch den Roboter erzeugt. Analyse erfolgte mit Hilfe von Text-Mining-Algorithmen, die eine regelbasierte Klassifikation von Fehlermeldungen, eine Ontologie/Taxonomie-Modellierung der Fehler und eine entsprechende Informationsvisualisierung beinhalteten

- Analyse von 144 Debugging-Protokollen mit Aktivitätsnamen, Datentypen, Variablenwerten, Argumenten usw. zur weiteren Abschätzung der Merkmalsausprägungen

Build II - Erstellung eines zweiten Prototypens für PEPA (PEPA 0.2) aufbauend auf den Evaluationsergebnissen

Evaluate III - Anwendung von PEPA 0.2 auf dieselben 16 Prozesse

- Durchführung von 11 semistrukturierten Experten-Interviews mit Anwendern von PEPA 0.2 (u. a. Junior SAP-Berater SD/ Geschäftsprozesse, SAP Key User/weltweite Projekte, Ingenieur Software-Prozesse und -Tools, Controller für den Geschäftsbereich Service)
Modell wurde für nicht geeignet befunden, Start des gemeinsamen Forschungsprojekts

Identifiziertes Verbesserungspotential für das Scoringmodell PEPA

PEPA 0.1 mit 12 Attributen in zwei Kategorien

Identifiziertes Verbesserungspotential für PEPA 0.1, insb. in Bezug auf die Ausgestaltung der Attribute sowie deren Bewertung

PEPA 0.2 mit 12 definierten Attributen mit klar abgegrenzten Merkmalsausprägungen in zwei Kategorien

Identifiziertes Verbesserungspotential für PEPA 0.2, insb. in Bezug auf die Gewichtung der Attribute 
Tab. 1 (Fortsetzung)

\begin{tabular}{|c|c|c|}
\hline $\begin{array}{l}\text { Entwick- } \\
\text { lungs- } \\
\text { schritt }\end{array}$ & Aktivitäten & Ergebnis \\
\hline Build III & $\begin{array}{l}\text { - Erstellung eines dritten Prototypens für PEPA (PEPA 0.3) } \\
\text { aufbauend auf den Evaluationsergebnissen }\end{array}$ & $\begin{array}{l}\text { PEPA } 0.3 \text { mit } 12 \\
\text { definierten Attribu- } \\
\text { ten mit klar abge- } \\
\text { grenzten Merkmals- } \\
\text { ausprägungen und } \\
\text { entsprechenden Ge- } \\
\text { wichtungen in zwei } \\
\text { Kategorien }\end{array}$ \\
\hline Evaluate IV & $\begin{aligned} \text { - Anwendung von PEPA } 0.3 \text { auf dieselben } 16 \text { Prozesse } \\
\text { - Durchführung von } 12 \text { semistrukturierten Experten-Interviews } \\
\text { mit Anwendern von PEPA } 0.3 \text { (u. a. Controller Geschäftsbe- } \\
\text { reich Services, Head of Finance/Controlling) }\end{aligned}$ & $\begin{array}{l}\text { Notwendige An- } \\
\text { passungen der At- } \\
\text { tributgewichte von } \\
\text { PEPA } 0.3\end{array}$ \\
\hline Build IV & - Anpassung der Attributgewichte und Erstellung von PEPA 1.0 & $\begin{array}{l}\text { PEPA } 1.0 \text { mit überar- } \\
\text { beiteten Attributge- } \\
\text { wichtungen }\end{array}$ \\
\hline
\end{tabular}

Das Unternehmen ist ein unabhängiges, mittelständisches Unternehmen in Familienbesitz, das ein nachhaltiges und kontinuierliches Wachstum in den Märkten der Zukunft anstrebt. Damit kann das Fallstudienunternehmen als typisches mittelständisches Unternehmen, wie es Daten in Deutschland häufig anzutreffen ist, bezeichnet werden. Parallel zur fortschreitenden Internationalisierung wurden die deutschen Standorte in den letzten Jahren systematisch weiterentwickelt. Hierbei setzt das Unternehmen überwiegend auf das ERP-System SAP ohne individuelle Programmierung oder Customizing. Diese Rahmenbedingungen wurden von uns bewusste gewählt, um eine größtmögliche Generalisierbarkeit erreichen zu können.

Sowohl die Investition in innovative Technologien als auch die damit verbundene zunehmende Vernetzung der Prozesse tragen dazu bei, dass die Forcierung von Prozessautomatisierung im Unternehmen zu einer strategischen Priorität geworden ist. Hier sollen durch den Einsatz von RPA neue Kapazitäten geschaffen werden, indem Mitarbeitern, die bisher mit der Sachbearbeitung betraut waren, mehr Freiraum gegeben wird und diese sich somit auf wertschöpfende Tätigkeiten konzentrieren können. Als Hauptschwächen wurden bereits die mangelnde Anpassung der Prozesse an die sich ändernden Anforderungen an die Fertigung im internationalen Kontext, die geringe Prozessqualität und die geringe Anpassungsfähigkeit der Prozesse festgestellt. Als vorrangiges Handlungsziel wurde anschließend die Reduzierung von Fehlerquellen definiert, was wiederum zu weniger Nachbearbeitung im Prozess und damit zu geringeren Prozesskosten führen soll.

Die Anwender rekrutieren sich aus direkt beteiligten RPA-Nutzern und RPAEntwicklern. Wir haben darauf geachtet, Benutzer und Entwickler nacheinander zu befragen, um im Interview auf ihre Ansichten eingehen zu können. Auch aus dem Gesichtspunkt der Vorerfahrung eignen sich die gewählten Anwender gut: fünf Teilnehmer haben eine Ausbildung zum operativen Prozessmanager, ein Teilnehmer eine Weiterbildung im Prozesscontrolling und die übrigen Teilnehmer eine Grundausbildung in Geschäftsprozessmanagement und „Control Objectives for Information and 
Related Technology“ durchlaufen. 7 Teilnehmer haben Erfahrung in der Entwicklung von RPA-Bots, wobei 3 Teilnehmer derzeit abteilungsübergreifend Roboter für das gesamte Unternehmen entwickeln.

\section{Entwicklung und Anwendung des Scoring-Modelles}

\subsection{Bewertungskriterien und -Attribute}

In der Praxis unterliegt die Auswahl der Bewertungskriterien naturgemäß der Subjektivität (Bhushan und Rai 2004). Die bisher verfügbaren Bewertungskriterien der Beratungsunternehmen waren im untersuchten Unternehmen zu ,diffus“ und ,nicht differenziert genug“ (Head of Finance and Controlling), zu ,,intransparent“ (Ingenieur Software-Prozesse und -Tools) sowie zu ,,abstrakt“ (Controller Geschäftsbereich Services). Für den Anwender stellte das bestehende Modell keine Entscheidungshilfe dar, ob der Prozess mithilfe von RPA, automatisierungsfähig ist.

Im Rahmen von Interviews wurden die Mitarbeiter zunächst unter Zusicherung von Anonymität nach ihrer persönlichen Einschätzung zu möglichen, wichtigen Kriterien befragt. Bereits in der ersten Befragungsrunde hatte sich ein Konsens der Befragten herauskristallisiert, auf welche Kriterien sich das Modell stützen sollte. Dabei zeigte sich, dass die Automatisierbarkeit und die wirtschaftlichen Auswirkungen für die Entscheidungsfindung von besonderer Bedeutung sind. Diese beiden Hauptkriterien der Automatisierungseignung und der wirtschaftlichen Auswirkungen wurden aus der ersten Interviewrunde herausgearbeitet und im Laufe der Entwicklung durch das DSR-Team und die Anwender weiter verfeinert. Dabei wurde das Hauptkriterium der Automatisierungseignung in drei neue Hauptkriterien (Ausschlusskriterien, Gütefaktoren und Stärkungskriterien) zerlegt. Ausgehend von diesen (in Summe vier) Hauptkriterien entwickelten sich im Laufe der Iterationszyklen 12 Bewertungskriterien. Ausgangslage war dabei eine Überprüfung der bisherigen Literatur (Yang et al. 2007; Bu und Xu 2009; McIvor 2008; Wang und Yang 2007; Wreford 2017; Lacity et al. 2011; Mahmoodzadeh et al. 2009) und in Summe 39 semi-strukturierte Interviews mit Mitarbeitern aus relevanten Abteilungen wie Controlling, Organisation, Personalwesen und Verwaltung. Auch die so verfeinerten Bewertungskriterien wurden in Gruppendiskussionen im Anschluss an jede Interviewrunde iterativ immer wieder voneinander abgegrenzt und so mit jedem Entwicklungszyklus eindeutiger definiert.

In Summe wurden so vier Hauptkriterien mit jeweils zwei bis vier Kriterien erarbeitet. Dabei werden die Hauptkriterien in ihrer Reihenfolge bearbeitet. Zuerst werden Prozesse auf vorliegende Ausschlusskriterien überprüft. Danach werden Kriterien erhoben und in das Modell eingepflegt, die die Effektivität und Effizienz der Automatisierung maßgeblich beeinflussen (Gütefaktoren). Als nächstes werden mit Stärkungskriterien Faktoren erfasst, die die Vorteile von RPA in den Prozessen verstärken können. Schließlich werden unter dem Punkt wirtschaftliche Auswirkungen, die bis dahin gesammelten Informationen über Kostenfaktoren und die damit verbundenen Kosteneffekte erfasst. Die einzelnen Kriterien werden im Folgenden detailliert vorgestellt: 
- Ausschlusskriterien sind Kriterien, welche zur effektiven Automatisierung zwingend notwendig sind

- Prozessart: RPA kann nur in Prozessen, die weitgehend regelbasiert sind, effektiv eingesetzt werden. Der Roboter ist nicht in der Lage, eigene Entscheidungen zu treffen oder zwischen zwei Alternativen abzuwägen.

- Datenquelle: Der Roboter arbeitet absolut fehlerfrei, solange die auszuwertenden Daten in der Computerumgebung zur Verfügung stehen. Dazu müssen die Daten in strukturierter Form vorliegen.

- Automatisierungsaufwand in anderen Systemen: Die Implementierung einer RPA-Automatisierung lohnt sich nur, wenn der Prozess nicht bereits in anderen Systemen automatisiert wurde. RPA als Brückenlösung ist ebenfalls möglich.

- Gütefaktoren sind Kriterien, welche die Effektivität und Effizienz der Automatisierung maßgeblich beeinflussen

- Datentyp: Der Roboter verfügt über eine vergleichsweise gute Bilderkennung, die jedoch nicht $100 \%$ ig genau ist. Systembrüche, wie das Lesen gescannter Dokumente, sind zu vermeiden.

- Stabilität: Der Software-Roboter arbeitet über die Benutzeroberfläche wie ein Mensch. Eine Veränderung dieser, z. B. durch Updates, muss vermieden werden, da sonst der Programmablauf des Roboters überarbeitet werden muss.

- Standardisierung: Ein standardisierter Prozess hilft bei der Umsetzung von RPAs und der Verbesserung der Prozesse im Allgemeinen. RPA stärkt den Standardisierungsprozess, indem immer das gleiche Verfahren angewendet wird. Dabei spielt es bei der Standardisierung keine Rolle, ob ein Prozess viele Varianten oder eine komplexe Ausführungstiefe hat. Hier ist die Existenz von durchgängigen, eindeutigen und systemisch abbildbaren Geschäftsregeln zur Identifizierung der richtigen Prozessvariante von primärer Bedeutung.

- Risiko: Prozesse, die im Falle eines Ausfalls des RPA-Systems nicht kritisch in den Betriebsablauf eingreifen, sind immer vorzuziehen.

- Stärkungskriterien verstärken die Vorteile von RPA

- Komplexität: Geeignete Prozesse können gestrafft werden und dürfen nicht zu viele verschiedene Varianten oder Zweige haben. Viele Ausführungsalternativen führen zu einem geringeren Grad der Automatisierungstauglichkeit.

- Fehleranfälligkeit (manuelle Bearbeitung): Wo Menschen Fehler machen, arbeitet der Roboter fehlerfrei, was die Qualität der Ergebnisse des automatisierten Prozesses verbessert.

- Beteiligte Anwendungen und Systeme: RPA ist überall dort sinnvoll, wo viele verschiedene Anwendungen und Systeme eingesetzt werden müssen, da hier keine Schnittstellen programmiert werden müssen.

- Wirtschaftliche Auswirkungen sind Kriterien, die zur monetären Bewertung eines Prozesses hinzugezogen werden

- Bearbeitungsdauer: Die Durchlaufzeit für die Bearbeitung des Prozesses. Je länger die Bearbeitungszeit, desto größer ist das Einsparpotenzial durch die Automatisierung. 
- Fallhäufigkeit: Die Häufigkeit der Prozessdurchführung in einem bestimmten Zeitraum. Je regelmäßiger ein Prozess vorkommt, desto größer ist das Einsparpotenzial durch die Automatisierung.

Im nächsten Schritt wurde für jedes der Kriterien Bewertungsskalen erzeugt. Diese wurden ebenfalls in den verschiedenen Build-/Evaluate-Zyklen schrittweise überarbeitet.

\subsection{Bewertungsskalen der Kriterien}

Die Bewertungskriterien kommen in der Praxis in unterschiedlicher Ausprägung vor. Diese Ausprägungen geben an, in wie fern ein Kriterium erfüllt ist. In den Build-/Evaluate-Zyklen hat es sich als vorteilhaft herausgestellt, die Extrempunkte der Skala zu beschreiben und dazwischen eine numerische Bewertungsskale mit 1 bis 5 Punkte einzuführen (Tab. 2). Die Leistungsbeurteilung der Mitarbeiter spiegeln die Extrempunkte in den Skalen wider. Bei der späteren Bewertung können Ermessensspielräume vorliegen, und somit empfiehlt es sich, die Punktvergabe durch ein Team durchzuführen.

Im Verlauf der Entwicklung von PEPA hat sich gezeigt, dass die Kriterien eine unterschiedlich hohe Gewichtung für die Einschätzung der Gesamteignung eines Prozesses für eine Automatisierung mit RPA haben. Diese Gewichtung wurde im letzten Schritt in mehreren Build-/Evaluate-Zyklen entwickelt.

\subsection{Gewichtung der Kriterien}

Nachdem die einzelnen Zielkriterien festgelegt worden waren, wurden diese gewichtet. Die Gewichtung zeigt dabei die relative Bedeutung der einzelnen Kriterien für die entsprechende Frage nach einer Automatisierungseignung oder den wirtschaftlichen Auswirkungen.

Zur Ermittlung der Gewichte wurden die verschiedenen Kriterien jeweils paarweise verglichen. Dieser Erhebungsprozess fand anonym und schriftlich in einem mehrstufigen Editierprozess statt, der teilweise mit Rückmeldungen der Befragten verbunden war. Die befragten Mitarbeitenden verglichen die verschiedenen Kriterien paarweise in mehreren Runden individuell mittels einer schriftlichen Umfrage. Dort wurde ebenfalls abgefragt, ob die Kriterien völlig unabhängig voneinander seien, um eine mögliche doppelte Gewichtung der Kriterien zu vermeiden. Als Diskussionsgrundlage erhielten die Mitarbeitenden dann die anonymisierten Gesamtergebnisse jeder Runde zur Kenntnisnahme. Diese Ergebnisse wurde für jedes Kriterium jeweils anhand einer Durchschnittswertberechnung ermittelt.

Daran schloss sich dann eine weitere Runde zur weiteren Diskussion, Klärung und Verfeinerung der Gewichtung an. Die unterschiedlichen Angaben der befragten Mitarbeiter hinsichtlich der individuellen Gewichtungen wurden dann einander gegenübergestellt. Nach drei Runden konnte die Spannweite der Gewichtungen im Konsensverfahren reduziert und verfeinert werden, sodass ein Endergebnis erzielt wurde. Da die Gewichte im DSR-Prozess empirisch ermittelt wurden, werden diese 
Tab. 2 Erklärung der Extremwerte der Bewertungsskalen

\begin{tabular}{lll}
\hline Kriterien & Bewertungsskalen & \\
\hline Ausschluss- & Entscheidungsbasiert - Die Prozesse erfor- & Regelbasiert - Strukturierter Pro- \\
kriterium: & dern mehr als 50\% manuelle Entscheidungs- & zessablauf, für den es wiederkehren- \\
Prozessart & findung, die durchlaufen werden müssen. Es & de Regeln und klare Handlungsan- \\
& gibt keine binären Entscheidungswege oder & weisungen gibt
\end{tabular}
die Bearbeitung durch einen Sachbearbeiter ist obligatorisch, weil keine Entscheidungsregeln gefunden werden können

Ausschluss- Unstrukturiert und analog - Mehr als 50\% der

kriterium:

Datenquelle im Prozess genutzten Datensätze hatten mindestens einen Fehler, der nicht durch Plausibilitätsprüfungen entdeckt werden kann. Mehr als $50 \%$ der im Prozess genutzten Datensätze müssen digitalisiert oder in ein anderes Format übertragen werden

Ausschluss- Niedrig - Abbildung des Prozesses im bekriterium: stehenden Workflow-Management-System Automatisierungsaufwand in anderen Systemen

Gütefaktor: Datentyp

Gütefaktor: Stabilität

Gütefaktor: Standardisierung

Gütefaktor: Risiko

Stärkungskriterium: Komplexität und Ausführung als modellierter Workflow möglich. Die Prozessabbildung ist unter Verwendung der Entscheidungslogik und der Verarbeitungslogik eines bestehenden Business Rule Management Systems (BRMS) möglich

Bilderkennung - Die erforderlichen Daten müssen zunächst durch Texterkennung aus verschiedenen Dokumenten, wie gescannten Papierdokumenten, PDF-Dateien oder digitalen Bildern, in Textinformationen umgewandelt werden

Häufige Updates - In der Vergangenheit wurde der Prozessablauf mindestens alle 8 Wochen angepasst, modifiziert, überarbeitet oder ergänzt

Gering - Es gibt mehr als 5 Prozessvarianten, die sich in ihrer Umsetzung erheblich unterscheiden. Die Auswahl der Prozessvariante basiert nicht auf einer zu definierenden Logik oder die Prozessdynamik lässt keine Standardisierung $\mathrm{zu}$

Hoch - Kritischer Geschäftsprozess, der bei einem Systemausfall nicht rechtzeitig aufrechterhalten oder wiederhergestellt werden kann

Komplex - Der Prozessfluss hat mehr als 5 Ausführungspfade, oder nicht alle verwendeten Ausführungspfade sind ausreichend erfasst und dokumentiert. Es ist nicht uneingeschränkt möglich, eine funktionale Logik für die Pfadauswahl zu definieren
Strukturiert und digital - Alle für den Prozessdurchführung benötigten Daten liegen in geordneter, strukturierter und plausibilisierter Form vor

Hoch - Bis dato existieren keine Workflow-Management-Systeme, Business Rule Management-Systeme oder integrierte Automatisierungslösungen im Unternehmen

Zahlenbasiert - Die Daten liegen vollständig in maschinenlesbaren und formatierten Formaten vor

Wenige Veränderungen - In der Vergangenheit gab es innerhalb von 24 Wochen keine Anpassungen oder Ergänzungen für mehr als einem Prozessschritt

Hoch - Standardmäßig wird der Prozess nur in einer Variante ausgeführt. Er ist vollständig dokumentiert und bereits optimiert

Gering - Geringe Kritikalität, mit einer maximal tolerierbaren Ausfallzeit von $48 \mathrm{~h}$ und unkritischen Wiederanlaufverfahren

Einfach - Der Prozessfluss ist vollständig modelliert und dokumentiert und folgt einfachen und transparent ohne Variationsmöglichkeiten der Abläufe.

Ein vollständiger und konsistenter Satz von Prozessregeln steht zur Verfügung 
Tab. 2 (Fortsetzung)

\begin{tabular}{|c|c|c|}
\hline Kriterien & Bewertungsskalen & \\
\hline $\begin{array}{l}\text { Stärkungs- } \\
\text { kriterium: } \\
\text { Fehleran- } \\
\text { fälligkeit } \\
\text { (manuelle } \\
\text { Bearbeitung) }\end{array}$ & $\begin{array}{l}\text { Gering - Geringe Fehleranfälligkeit (weniger } \\
\text { als } 1 \text { Fehler in } 1000 \text { Prozessdurchläufen), z.B. } \\
\text { durch robustes Benutzerkonzept, unterstüt- } \\
\text { zende Benutzeroberflächen oder vorhandene } \\
\text { Plausibilitätsprüfungen }\end{array}$ & $\begin{array}{l}\text { Hoch - Mehr als } 5 \text { Fehler pro } 100 \\
\text { Prozessausführungen, die auf } \\
\text { menschliche Faktoren wie fehler- } \\
\text { hafte Datenübertragung und Benut- } \\
\text { zerfehler zurückzuführen sind }\end{array}$ \\
\hline $\begin{array}{l}\text { Stärkungs- } \\
\text { kriterium: } \\
\text { Beteiligte } \\
\text { Anwen- } \\
\text { dungen \& } \\
\text { Systeme }\end{array}$ & Wenige - Nur ein beteiligtes System & $\begin{array}{l}\text { Viele - Mehr als } 4 \text { Systeme oder } \\
\text { Medienbrüche in primären Pro- } \\
\text { zessen und sekundäre Prozesse im } \\
\text { ERP-System und anderen Unterneh- } \\
\text { mensinformationssystemen }\end{array}$ \\
\hline $\begin{array}{l}\text { Wirtschaftliche } \\
\text { Auswirkung: } \\
\text { Bearbei- } \\
\text { tungsdauer }\end{array}$ & $\begin{array}{l}\text { Gering - Der Prozess erfordert } 7 \% \text { der mo- } \\
\text { natlichen Arbeitszeit pro Prozessverantwortli- } \\
\text { chen }\end{array}$ & $\begin{array}{l}\text { Hoch - Der Prozess erfordert mehr } \\
\text { als } 20 \% \text { der monatlichen Arbeitszeit } \\
\text { pro Prozessverantwortlichem }\end{array}$ \\
\hline $\begin{array}{l}\text { Wirtschaftliche } \\
\text { Auswirkung: } \\
\text { Fallhäufig- } \\
\text { keit }\end{array}$ & $\begin{array}{l}\text { Selten - Unter } 4 \text { Prozessdurchführungen pro } \\
\text { Monat }\end{array}$ & $\begin{array}{l}\text { Oft - Über } 20 \text { Prozessdurchführun- } \\
\text { gen pro Monat }\end{array}$ \\
\hline
\end{tabular}

abschließend auf 100 normiert, um einen Gesamteignungsgrad in Prozent zu erhalten.

In der Version 0.3 des vorgestellten PEPA-Modells konnte demnach in der letzten Iterationsstufe für jeden Prozess durch Bestimmung der Eigenschaften der Attribute festgestellt werden, inwieweit der jeweilige Prozess das jeweilige Kriterium erfüllt. Durch Multiplikation des Erfüllungsgrades eines Attributs mit den in Version 0.3 für die jeweilige Organisation definierten Gewichtungsfaktoren kann dann für jeden untersuchten Prozess die Automatisierungseignung bzw. die wirtschaftlichen Auswirkungen ermittelt werden.

\subsection{Gesamtmodell und Anwendung}

Zur Vereinfachung der Anwendung von PEPA wurden die dargestellten Überlegungen in Bezug auf Kriterien, Bewertungsskalen und Gewichtungen in ein SoftwareTool überführt. Dieses Tool ermöglicht die graphische Eingabe der Einschätzungen je Prozess und berechnet dann automatisiert die Gesamteignung in Prozent (vgl. Abb. 3).

Bei der Anwendung von PEPA in einer Organisation werden typischerweise fünf Schritte durchlaufen. Zuerst werden einmalig die Gewichtungen der Kriterien angepasst. In ersten Diskussionen und Anwendungen von PEPA mit weiteren Organisationen stellte sich heraus, dass die Priorität der Kriterien je nach Lage der Organisation unterschiedlich ist. Als nächstes müssen für jeden Prozess die Kriterien bewertet werden. Dazu dienen die in Abschn. 5.1 und 5.2 dargestellten Beschreibungen und Skalierungen. Das Modell berechnet dann drittens automatisch ein Gesamtergebnis je Prozess (vgl. Abb. 4) und bildet darauf aufbauend viertens einen Entscheidungs- 


\begin{tabular}{|c|c|c|c|c|c|c|c|}
\hline \multirow{2}{*}{$\begin{array}{l}\text { Kriterium } \\
\text { Ausschlusskriterium }\end{array}$} & \multicolumn{7}{|c|}{ Bewertungsskala } \\
\hline & & 1 & 2 & 3 & 4 & 5 & \\
\hline Prozessart & entscheidungsbasiert & c & $c$ & $r$ & $c$ & $c$ & regelbasiert \\
\hline Datenquelle & unstrukturiert und analog & $c$ & $c$ & $c$ & $c$ & $c$ & strukturiert und digital \\
\hline $\begin{array}{l}\text { Automatisierungsaufwand in anderen } \\
\text { Systemen }\end{array}$ & niedrig & $c$ & $c$ & $c$ & $c$ & $c$ & hoch \\
\hline Gütefaktor & & 1 & 2 & 3 & 4 & 5 & \\
\hline Datentyp & Bilderkennung & $c$ & $c$ & $c$ & $c$ & $c$ & zahlenbasiert \\
\hline Stabilität & häufige Updates & $c$ & $c$ & $c$ & $c$ & $c$ & wenige Veränderungen \\
\hline Standardisierung & gering & $c$ & $c$ & $c$ & $c$ & $c$ & hoch \\
\hline Risiko & hoch & $c$ & $c$ & $c$ & $c$ & $c$ & gering \\
\hline Stärkungskriteriumn & & 1 & 2 & 3 & 4 & 5 & \\
\hline Komplexität & komplex & $c$ & $c$ & $c$ & $c$ & $c$ & einfach \\
\hline Fehleranfalligkeit (man. Bearbeitung) & gering & $r$ & $r$ & $c$ & $c$ & $r$ & hoch \\
\hline Beteiligte Anwendungen \& Systeme & wenige & $c$ & $c$ & $c$ & $c$ & $c$ & viele \\
\hline Wirtschaftliche Auswirkung & & 1 & 2 & 3 & 4 & 5 & \\
\hline Bearbeitungsdauer & gering & $c$ & $c$ & $c$ & $c$ & $c$ & hoch \\
\hline Fallhäufigkeit & selten & $c$ & $c$ & $c$ & $c$ & $c$ & oft \\
\hline
\end{tabular}

Abb. 3 Grafische Benutzeroberfläche des Scoring-Modells zur Prozessauswahl für einen RPA-Einsatz

vorschlag in Form einer Rangfolge der einzelnen Prozesse. Als letztes muss dieser Entscheidungsvorschlag diskutiert und verabschiedet werden.

\section{Zusammenfassung, Fazit und Ausblick}

Dieser Artikel stellt die Ergebnisse eines DSR-Projekts zur Entwicklung des Scoring-Modells PEPA für die Eignung von Prozessen für die Automatisierung mittels RPA dar. Ursprünglich baut PEPA auf existierenden, in der Praxis verwendeten Modellen sowie wissenschaftlicher Literatur zu RPA auf. In vier Build-/EvaluateZyklen wurde das Modell dann schrittweise bei einem Fallstudienpartner aus der Industrie verfeinert.

Die Vorteile der Anwendung von PEPA für das Fallstudienunternehmen lagen zunächst in den formalen Entscheidungsstrukturen und der standardisierten Kommunikation über die ausgewählten Prozesse. Die Anwendung von PEPA bedeutete jedoch zunächst auch einen höheren Aufwand, da die benötigten Informationen zwar vorhanden waren, aber gesammelt und geordnet werden mussten. Durch die Anwendung bei dem Fallstudienunternehmen konnte sich aber trotzdem zeigen, dass die Rolle von PEPA in der Praxisanwendung eher für RPA-Projekte mit geringeren Budgets relevant ist, bei denen bisher wenig oder keine strukturierte Entscheidungsfindung verwendet wurde. Das hier vorgestellte Scoring-Modell unterstützt dabei 
Abb. 4 Beispielhafte Darstellung der Ergebnisse in der Benutzeroberfläche des Bewertungsmodells

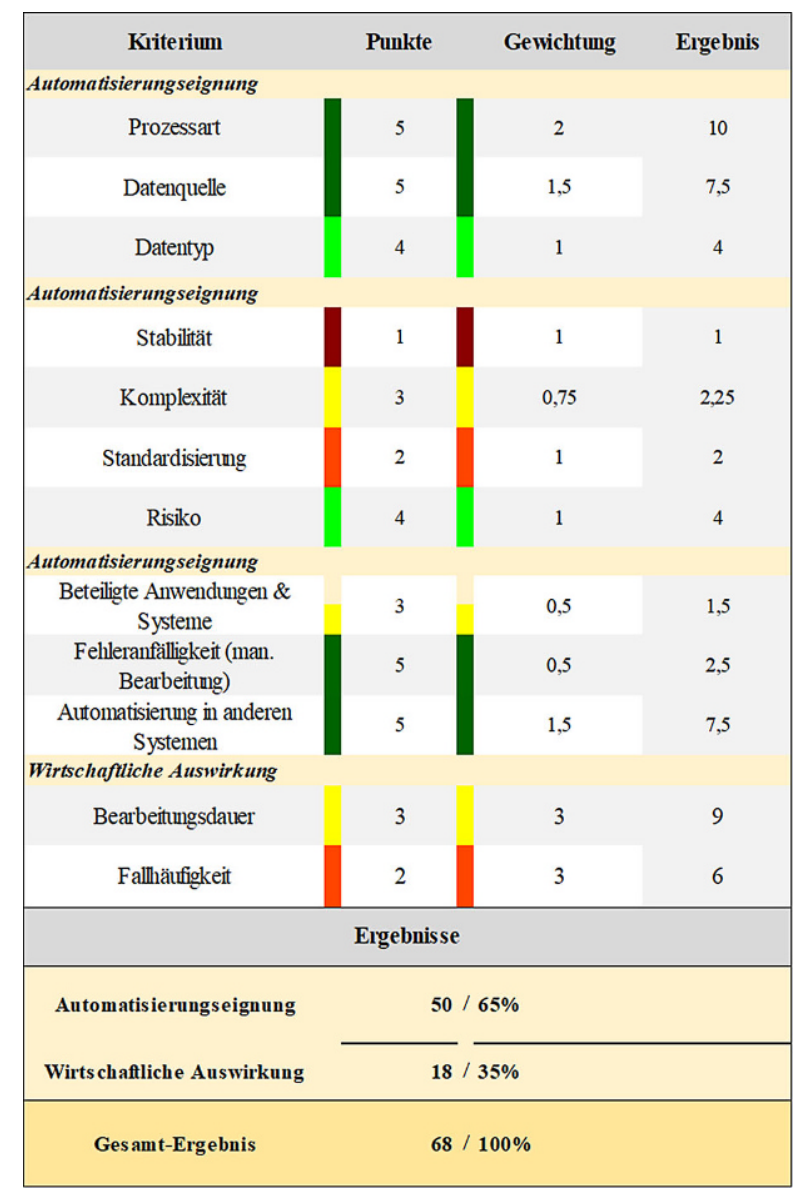

in der Praxis die Unternehmensführung zusätzlich zu den traditionellen finanziellen Methoden des Entscheidungsfindungsprozesses.

Durch die Anwendung beim in Bezug auf Größe und eingesetzte Kernsysteme durchaus als typisch für den deutschen Mittelstand zu bezeichnenden Fallstudienunternehmen konnte das Bewertungsmodell evaluiert werden. Um eine erste Generalisierung über diesen ,typischen“ deutschen Mittelstand hinaus zu untersuchen wurde PEPA zusätzlich erfolgreich auf 7 Verwaltungsprozesse einer deutschen Kommunalverwaltung angewendet. Sowohl in Bezug auf Organisationskultur und Größe als auch auf eingesetzte IT-Systeme konnte eine große Kontrastierung zum eigentlichen Fallstudienunternehmen realisiert werden. Die Zielrichtung beider Organisationen, Prozesse untereinander in Bezug auf ihre Eignung für einen RPA-Einsatz zu vergleichen und zu priorisieren wurde dabei stets erfüllt. Besonders bemerkenswert ist, dass in beiden Organisationen die formalen und informellen Entscheidungsprozesse, die in der Vergangenheit für die Auswahl solcher Projekte verwendet wurden, zum ersten Mal vollständig erfasst wurden. Allerdings wurden bei der Anwendung die Gewichte der Kriterien branchenspezifisch angepasst. 
Aus der Evaluation in beiden Organisationen konnten die folgenden Vor- und Nachteile von PEPA herausgearbeitet werden. Erstens bietet PEPA einen konsistenten Überblick über den Auswahlprozess und über die Metriken, die die Priorisierungsentscheidung beeinflussen. Zweitens ermöglicht PEPA einen systematischen Ansatz der Informationssammlung und Auswertung über verschiedene Attribute und Ebenen. Dadurch wurde neben der reinen Priorisierungsentscheidung in vielen Fällen auch eine Vielzahl neuer Informationen über den tatsächlichen Prozessablauf hervorgebracht. Drittens konnten die Fallstudienorganisationen durch PEPA die Auswahl bestimmter Prozesse begründen und haben im Verlauf der RPA-Implementierungsprojekte auch mit Verweis auf PEPA an den Entscheidungen festgehalten. Viertens konnten keine logischen Fehler oder intransparenten Priorisierungen identifiziert werden. Hierzu wurden alle in der Praxis generierten Ergebnisse des Modells von den jeweiligen Anwendern und Experten und anschließend vom Forschungsteam gegengeprüft. Alle untersuchten Prozessgrößen konnten in der erstellten Priorisierungssequenz rekonstruiert und als inhaltlich und formal korrekt eingestuft werden.

PEPA ist insbesondere durch diese Entwicklung mit der Praxis geeignet, auch auf andere Unternehmen transferiert zu werden. Es ermöglicht so Praktikern verschiedener Branchen eine schnelle Priorisierung von Prozessen für die RPA-Automatisierung und spart so trotz kurzfristig höherer Priorisierungsaufwände mittelfristig Kosten durch Vermeidung der Automatisierung von niedrig-prioren Prozessen.

Nichtsdestotrotz ist die Anwendung von PEPA zurzeit noch limitiert. Zum einen ist die Objektivität der Ergebnisse von PEPA als Scoring-Modell eingeschränkt. PEPA beruht auf einer Auswahl von nicht-metrisch skalierten Kriterien und Gewichten, die gegebenenfalls eine Scheinobjektivität erzeugen. In der Anwendung müssen also getroffene Annahmen für Attributsausprägungen hinterfragt werden. Selbst in der letzten Iteration des zugrundeliegenden Modells konnten subjektive Einflüsse der Anwender während der Bewertung der Kriterien nicht immer vollständig transparent dargestellt werden. Um diese Nachteile zu verringern, sollen im weiteren Verlauf unserer Forschung korrelierende Plausibilitätsbeziehungen aus Vergangenheitswerten entwickelt werden, die es ermöglichen sollen, Inkonsistenzen in der Bewertung zu erkennen und zu beseitigen.

Zum anderen ist das Modell zwar aufbauend auf wissenschaftlichen Publikationen in enger Zusammenarbeit mit einem Praxisunternehmen entwickelt und in einer zweiten Organisation angewendet worden, ein skalierter Test über die beiden Organisationen hinaus steht allerdings noch aus. Um eine weitere, größere Verallgemeinerbarkeit zu gewährleisten, müssen Evaluierungen, auch über längere Zeiträume, in Zukunft gemeinsam mit anderen, insbesondere von den betrieblichen Gegebenheiten abweichenden Unternehmen durchgeführt werden.

Zukünftige Forschung könnte sich auch mit der Anpassung des Modells auf eine stärkere Integration von Cognitive Automation und Künstlicher Intelligenz (KI) in RPA befassen. So kann ein Zusammenwachsen von RPA mit diesen fortgeschrittenen Methoden der Prozessautomatisierung beobachtet werden (Kannan 2018). Beispielsweise kann RPA dann Methoden zur Zeichenerkennung (Optical Character Recognition) einsetzen, um auch aus unstrukturierten Bild-Daten strukturierte Informationen abzuleiten. In diesem Fall wird sich möglicherweise die Gewichtung des Gütefaktors Datentyp verändern und der Faktor weniger stark in die Gesamtpriori- 
sierung einwirken. Ähnliche Verschiebungen von Gewichtungen oder Extremwerten der Kriterien können sich auch durch die Integration von anderen fortgeschrittenen und KI-näheren Methoden ergeben.

Abschließend ist das Modell zwar ein Schritt, die von van der Aalst und Kollegen aufgeworfene Forschungslücke zu schließen (van der Aalst et al. 2018b). Hierzu benötigt es jedoch noch weiterer anwendungsorientierter empirischer Forschung, um eine Allgemeingültigkeit des Modells auch für andere Industrien und Branchen sicherzustellen. Außerdem können die verwendeten Gewichte einer weiteren Untersuchung unterzogen werden. Hierzu würden sich beispielsweise Delphi-Studien oder quantitative Umfragen anbieten.

Danksagung Die Autoren danken dem Praxispartner, mit dem dieses anwendungsorientierte Forschungsprojekt durchgeführt wurde, sowie den studentischen Mitarbeitern, die bei der Datenerhebung und -Auswertung unterstützt haben.

Funding Open Access funding enabled and organized by Projekt DEAL.

Open Access Dieser Artikel wird unter der Creative Commons Namensnennung 4.0 International Lizenz veröffentlicht, welche die Nutzung, Vervielfältigung, Bearbeitung, Verbreitung und Wiedergabe in jeglichem Medium und Format erlaubt, sofern Sie den/die ursprünglichen Autor(en) und die Quelle ordnungsgemäß nennen, einen Link zur Creative Commons Lizenz beifügen und angeben, ob Änderungen vorgenommen wurden.

Die in diesem Artikel enthaltenen Bilder und sonstiges Drittmaterial unterliegen ebenfalls der genannten Creative Commons Lizenz, sofern sich aus der Abbildungslegende nichts anderes ergibt. Sofern das betreffende Material nicht unter der genannten Creative Commons Lizenz steht und die betreffende Handlung nicht nach gesetzlichen Vorschriften erlaubt ist, ist für die oben aufgeführten Weiterverwendungen des Materials die Einwilligung des jeweiligen Rechteinhabers einzuholen.

Weitere Details zur Lizenz entnehmen Sie bitte der Lizenzinformation auf http://creativecommons.org/ licenses/by/4.0/deed.de.

\section{Literatur}

van der Aalst WMP, Becker J, Bichler M, Buhl HU, Dibbern J, Frank U et al (2018a) Views on the past, present, and future of business and information systems engineering. Bus Inf Syst Eng 60(6):443-477. https://doi.org/10.1007/s12599-018-0561-1

van der Aalst WMP, Bichler M, Heinzl A (2018b) Robotic process automation. Bus Inf Syst Eng 60(4):269-272. https://doi.org/10.1007/s12599-018-0542-4

Asatiani A, Penttinen E (2016) Turning robotic process automation into commercial success. J Inf Technol Teach Cases 2:67-74

Becker F, Meyer M, Redlich B, Siemon D, Lattemann C (2020) Open KMU: Mit Action Design Research und Design Thinking gemeinsam innovieren. HMD 57:274-284. https://doi.org/10.1365/s40702020-00604-z

Becker J, Knackstedt R, Pöppelbuß J (2009) Developing maturity models for IT management. Bus Inf Syst Eng 3:213-222. https://doi.org/10.1007/s12599-009-0044-5

Bensberg F (2019) Nutzwertanalyse. Hg. v. Norbert Gronau, Jörg Becker, Natalia Kliewer, Jan Marco Leimeister und Sven Overhage. Berlin (Enzyklopädie der Wirtschaftsinformatik - Online-Lexikon. https://www.enzyklopaedie-der-wirtschaftsinformatik.de. Zugegriffen: 30. März 2020 (zuletzt aktualisiert am 27.02.2019)

Bhushan N, Rai K (2004) Strategic decision making: applying the analytic hierarchy process. Springer, Berlin 
Bu X, Xu J (2009) Developing a decision model for it outsourcing using analytic hierarchy process. In: 2009 International Conference on Management and Service Science, S 1-5 https://doi.org/10.1109/ ICMSS.2009.5305800

Czarnecki C, Bensberg F, Auth G (2019) Die Rolle von Softwarerobotern für die zukünftige Arbeitswelt. HMD 56(4):795-808. https://doi.org/10.1365/s40702-019-00548-z

Dumas M, La Rosa M, Mendling J, Reijers HA (2018) Fundamentals of business process management, 2. Aufl. Springer, Berlin, Heidelberg

Gerogiannis VC, Fitsilis P, Voulgaridou D, Kirytopoulos KA, Sachini E (2010) A case study for project and portfolio management information system selection: a group AHP-scoring model approach. Int J Proj Organ Manag 2(4):361-381. https://doi.org/10.1504/IJPOM.2010.035873

Hevner AR, March ST, Park J, Ram S (2004) Design science in information systems research. MISQ 28(1):75-105

Hofmann J, Günther J (2019) Arbeiten 4.0 - Eine Einführung. HMD 56(4):687-705. https://doi.org/10. 1365/s40702-019-00553-2

Jimenez-Ramirez A, Reijers HA, Barba I, Del Valle C (2019) A method to improve the early stages of the robotic process automation lifecycle. In: International Conference on Advanced Information Systems Engineering, S 446-461

Kannan V (2018) When RPA met AI: the rise of cognitive automation. https://www.uipath.com/blog/whenrpa-met-ai. Zugegriffen: 20. Okt. 2019

Lacity MC, Willcocks LP (2016) Robotic process automation at Telefónica O2. MISQE 15(1):21-35

Lacity MC, Solomon S, Yan A, Willcocks LP (2011) Business process outsourcing studies: a critical review and research directions. J Inf Technol 26(4):221-258. https://doi.org/10.1057/jit.2011.25

Mahmoodzadeh E, Jalalinia S, Nekui Yazdi F (2009) A business process outsourcing framework based on business process management and knowledge management. Bus Proc Manag J 15(6):845-864. https://doi.org/10.1108/14637150911003748

Manyika J, Lund S, Chui M, Bughin J, Woetzel J, Batra P et al (2017) Jobs lost, jobs gained: workforce transitions in a time of automation. A McKinsey Global Institute report. McKinsey Global Institute, (Hg. v. McKinsey \& Company), San Francisco

McIvor R (2008) What is the right outsourcing strategy for your process? Eur Manag J 26(1):24-34. https:// doi.org/10.1016/j.emj.2007.08.008

Meier C, Seufert S, Guggemos J (2019) Arbeitswelt 4.0 und Smart Machines: Augmentation als Herausforderung für die Personalentwicklung. HMD 56(4):823-839. https://doi.org/10.1365/s40702-01900552-3

Mendling J, Decker G, Hull R, Reijers HA, Weber I (2018) How do machine learning, robotic process automation, and blockchains affect the human factor in business process management? CAIS 43:297-320. https://doi.org/10.17705/1CAIS.04319

Mettler T (2011) Maturity assessment models: a design science research approach. IJSSS 3(1/2):81-98

Mohapatra S (2009) Business process automation, 1. Aufl. PHI Learning, New Delhi

Muralidhar K, Santhanam R, Wilson RL (1990) Using the analytic hierarchy process for information system project selection. Inf Manag 18(2):87-95. https://doi.org/10.1016/0378-7206(90)90055-M

Niesen T, Houy C, Fettke P (2019) Digitale Transformation von Prozessen in der Rechtsberatung: Anwendungsszenarien im Steuerbereich am Beispiel des ARGUMENTUM-Systems. HMD 56(4):766-779. https://doi.org/10.1365/S40702-019-00544-3

Øvrelid E, Halvorsen MR (2018) Process innovation with lightweight IT at an emergency unit. In: Proceedings of the 51st Hawaii International Conference on System Sciences

Penttinen E, Kasslin H, Asatiani A (2018) How to choose between robotic process automation and backend system automation? In: Proceedings of the 26th European Conference on Information Systems Portsmouth, UK (https://aisel.aisnet.org/ecis2018_rp/66)

Plattfaut R (2019) Robotic process automation-process optimization on steroids? In: Krcmar H, Fedorowicz J, Boh WF, Leimeister JM, Wattal S (Hrsg) Development Dynamics of Digital Infrastructure and Organization: The Case of Global Payments Innovation. Proceedings of the 40th International Conference Munich (AIS Electronic Library. Online verfügbar unter https://aisel.aisnet.org/icis2019/ practicel_is $\_$research/practicel_is $\backslash$ research $/ 3$ )

Poeppelbuss J, Niehaves B, Simons A, Becker J (2011) Maturity models in information systems research: literature search and analysis. CAIS 29:505-532. https://doi.org/10.17705/1CAIS.02927

Sein, Henfridsson, Purao, Rossi, Lindgren (2011) Action Design Research. MIS Quarterly 35(1):37

Sumner M (2005) Enterprise resource planning, 1. Aufl. Pearson/Prentice Hall, Upper Saddle River, NJ

Syed R, Suriadi S, Adams M, Bandara W, Leemans SJJ, Ouyang C et al (2020) Robotic process automation: contemporary themes and challenges. Comput Ind 115:1-15 
Wang J-J, Yang D-L (2007) Using a hybrid multi-criteria decision aid method for information systems outsourcing. Comput Oper Res 34(12):3691-3700. https://doi.org/10.1016/j.cor.2006.01.017

Wei CC, Chien C-F, Wang M-JS (2005) An AHP-based approach to ERP system selection. Int J Prod Econ 96(1):47-62. https://doi.org/10.1016/j.ijpe.2004.03.004

Wreford JW (2017) Critical success factors in the offshore business process outsourcing of debt collection to India. Doctoral Thesis. Curtin University. https:/espace.curtin.edu.au/handle/20.500.11937/68366 Zugegriffen am: 12.10.2020

Yang D-H, Kim S, Nam C, Min J-W (2007) Developing a decision model for business process outsourcing. Comput Oper Res 34(12):3769-3778. https://doi.org/10.1016/j.cor.2006.01.012 